\title{
A new compact model for the analysis of the anomalies in I-V characteristics of Schottky diodes
}

\author{
J. Racko, D. Donoval and V. Drobny \\ Department of Microelectronics, Slovak Technical University in Bratislava, \\ Ilkovicova 3, SK-812 19 Bratislava, Slovakia \\ phone:+421765423486 fax:+421765423480 e-mail:donoval@elf.stuba.sk
}

\begin{abstract}
A new enhanced analytical model including quantum-mechanical effects which reflects the Internal behoviour of Schotiky structures more adequately and corresponds to experimental characteristics is presented. The contribution of surface generation - recombination current to the total current through the interface is significant, parficularly for low forward applied voltages and low temperatures and cannot be neglected in the model for parameters extractlon.
\end{abstract}

\section{Introduction}

Parameters extraction based on the good agreement between experimental and simulated results can provide a valuable aid for design optimisation and predictive analysis of novel semiconductor structures and their properties. Although the Schottky structures play an important role among semiconductor structures [1] the analysis of measured characteristics and extraction of their parameters is not unique and is limited by inadequate understanding of the nature of their electrical behaviour. Lateral barrier height distribution, different modified Richardson constants for metal and semiconductor, and the influence of an interfacial isolation layer with interface states are usually considered as the origin of the anomalous behaviour of I-V characteristics of Schottky diodes [2-6]. However, the contribution of other mechanisms of current flow through the metal-semiconductor interface such as bulk and surface generation-recombination, tunnelling and leakage current at the contact periphery can cause significant deviations from the ideal thermionic emission-diffusion theory [7]. Assuming the sum of all above mentioned components the selected parameters $\left(\phi_{b}, n\right)$ are evaluated with higher precision from I-V curves in a wide temperature range [8]. The main disadvantage of such an empirical approach is that it neglects the interactions between the individual current flow mechanisms.

\section{Our approach}

We present a refined approach leading to a compact model which includes all the above mentioned mechanisms and their mutual interactions. The solution leads to a lengthy formula 


$$
J=q \frac{v_{d}\left(v_{t b}^{s}+v_{s}\right)}{v_{d}+v_{t a}^{s}+v_{s}}\left[n_{s} \exp \left(\frac{q V_{a}}{k T}\right)-n_{M} \frac{v_{t}^{M}+v_{s}^{0}}{v_{t s}^{s}+v_{s}}+\frac{J_{t}\left(x_{m}\right)}{q\left(v_{t c}^{s}+v_{s}\right)}+n_{t}+n_{g r}\right]
$$

where $v_{d}$ is the drift-diffusion velocity

$$
\left(v_{d}\right)^{-1}=\int_{x_{m}}^{w} \frac{1}{D_{n}(x)} \exp \left(-\frac{q \psi(x)}{k T}\right) d x
$$

and $\left(v_{l e}+v_{s}\right)$ is the total recombination velocity with

$$
v_{t \omega}=\frac{A_{s}^{* *} T^{2}}{q N_{c}} \exp \left(\frac{\phi_{b}}{k T}\right) \int_{\phi_{b} / k T}^{\infty} T_{m}^{s M}(\varepsilon) \exp \left(-\frac{\varepsilon}{k T}\right) \frac{d \varepsilon}{k T}
$$

and

$$
v_{s}=\frac{\sigma_{n} \sigma_{p} v_{t h} N_{t s} p_{s}}{\sigma_{n}\left(n_{s} \exp \left(\frac{V_{a}}{V_{t}}\right)+n_{i} \exp \left(\frac{E_{t s}-E_{i}}{k T}\right)\right)+\sigma_{p}\left(p_{s}+n_{i} \exp \left(\frac{E_{i}-E_{t}}{k T}\right)\right)}
$$

Equation (4) is based on the Shockley-Hall-Reed model and characterises the surface recombination velocity with $N_{t s}$ and $E_{t s}$ as density and energy of dominating surface traps, respectively. The last three terms in eq (1) characterise the tunnelling and bulk generation recombination mechanism [9]

$$
\begin{gathered}
J_{t}(x)=\frac{T}{k}\left[A_{s} \int_{s_{\min }}^{s(x)} F(\varepsilon) T_{r}^{s M}(\varepsilon)\left(1-F^{0}(\varepsilon)\right) d \varepsilon-A_{M} \int_{s_{\min }}^{s(x)} F^{0}(\varepsilon) T_{r}^{M S}(\varepsilon)(1-F(\varepsilon)) d \varepsilon\right] \\
n_{t}=\int_{x_{m}}^{x_{m}} \frac{J_{t}(x)}{q D_{n}(x)} \exp \left(-\frac{q \psi(x)}{k T}\right) d x \quad n_{g_{r}}=\int_{x_{m}}^{w}\left[\frac{1}{q D_{n}(x)} \exp \left(-\frac{q \psi(x)}{k T}\right) \int_{x_{m}}^{x} U\left(x^{\prime}\right) d x^{\prime}\right] d x
\end{gathered}
$$

All other coefficients have their usual meaning.

\section{Experimental results and discussion}

There is a significant deviation between experimental and simulated I-V characteristics based on the pure thermionic emission - diffusion theory at low forward bias and low temperature (Fig. 1). A dependence of velocity on applied voltage is shown in Fig. 2. At low temperature $(\mathrm{T}=206 \mathrm{~K})$ for low forward applied voltage (region I) $v_{t e}+v_{s}>v_{d}$ and the drift-diffusion velocity limits the total current. While the transport of free carriers through the space charge region is the bottleneck for current flow at low temperature, the value of $v_{s}$ is negligible in comparison with $v_{t e}$ at room temperature $(T=300 \mathrm{~K})$, region 1 disappears 


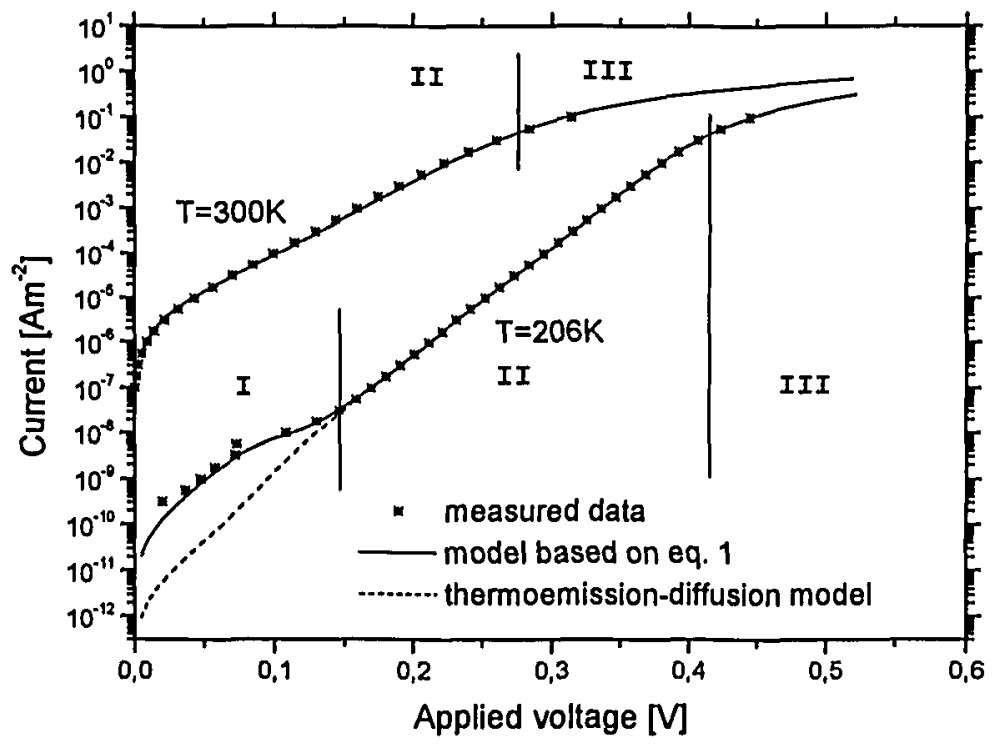

Fig. 1. Experimental and simulated I-V characteristics

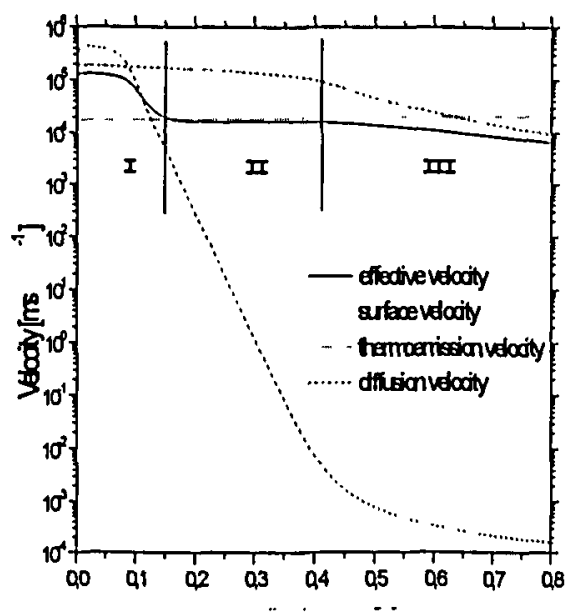

a)

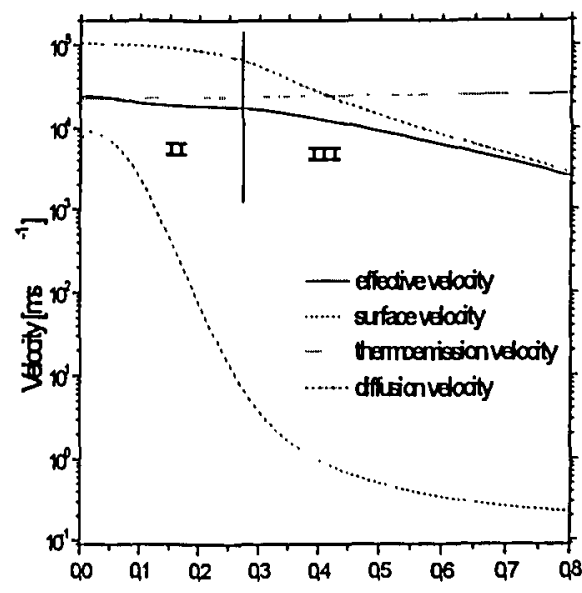

b)

Fig. 2 The simulated values of velocity as a function of applied voltage for a) $\mathrm{T}=206 \mathrm{~K}$ and b) $\mathrm{T}=300 \mathrm{~K}$

(Fig. 2b) and the transport through the interface limits the total current. As can be clearly seen from Fig. 1, the corresponding region I is missing for I-V characteristics at $300 \mathrm{~K}$ where both experimental and simulated characteristics are almost identical. The $v_{s}$ decreases 
exponentially with $V_{a}$ in both cases and therefore $v_{t e}+v_{s}<v_{d}$ in region II ( $V_{a}$ medium) and the total current is limited by the thermionic emission through the interface. In region III ( $V_{a}$ large) $v_{d}$ decreases with $V_{a}$ and the total current again becomes limited by the transport of free carriers towards the interface. The best correlation between the experimental and simulated characteristics was obtained for $N_{t s}=4 \times 10^{18} \mathrm{~m}^{-2}, E_{t s}=E_{t}$ and temperature dependent electron and hole capture cross section [10]

$$
\sigma=\sigma_{0} \exp \left(-\frac{\Delta E}{k T}\right) d x
$$

with $\sigma(206)=1 \times 10^{-18} \mathrm{~m}^{-2}$ and $\sigma(300)=1.5 \times 10^{-19} \mathrm{~m}^{-2}$.

\section{Conclusion}

The presented model represents a reasonable compromise between physical rigorosity and its practical applicability for parameters extraction based on the improved correlation of experimental and simulated I-V characteristics. The implementation of non-standard and quantum mechanical effects into the compact analytical model for current flow is increasingly important for Schottky structures with shrinking dimensions. Parameters extraction based on advanced, more complex physical models contributes considerably to the analysis of the origin of device anomalies and thus to characterising the fabrication process of device preparation .

Acknowledgement - This work was supported by grant No. 95/5195/286 of the Slovak Ministry of Education.

\section{References}

[1] B.J. Baliga, IEEE Transactions on Electron Devices, 43, p. 1717, (1996)

[2] R.T. Tung, Physical Review Letters, 66, p.72, (1991)

[3] J. Osvald, Solid St. Electron, 35, p. 1629, (1992)

[4] M. Missous and E.H. Rhoderick, J. Appl.Phys., 69, (10),7142, (1991)

[5] Z.J. Horvath, Vacuum, 46, p. 963, (1995)

[6] D.Schroder, Modelling of Interface Current Transport for Device Simulation, Springer Verlag, Wien, New York 1994

[7] D. Donoval, M. Barus and M. Zdimal, Solid St. Electron., 32, p. 1365, (1991)

[8] D. Donoval, V. Drobny and M. Luza, A Contribution to the Analysis of the I-V Characteristics of Schottky Diodes, Accepted in Solid St. Electron., (1998)

[9] J. Racko, Integral Transport Theory of Current Flow through Metal-Semiconductor-Metal Structures, PhD Thesis, (in Slovak), FEI STU Bratislava, 1994

[10] C.H. Henry and D.V. Lang, Phys. Rev. B, 15, p. 989, 1977 